\title{
Expression of a quantitative character radius incompletus, temperature effects, and localization of a mobile genetic element Dm-412 in Drosophila melanogaster
}

\author{
L.A. VASILYEVA, S.A. ZABANOV, V.A. RATNER, I.F. ZHIMULEV, \\ M.O. PROTOPOPOV and E.S. BELYAEVA \\ Institute of Cytology and Genetics, \\ Academy of Sciences of the USSR, Siberian Branch, \\ Novosibirsk 630090, USSR
}

\begin{abstract}
Summary
The radius incompletus mutation (ri) causes a gap in the radial wing vein, $L 2$. A control line ( $r i C$ ) of the mutation $r i$ was selected for increase and decrease of radial vein. In the riSN strain, extreme expression of this quantitative character is almost complete elimination of $L 2$ (negative selection : $S N$ ). In the riSP strain, the $L 2$ vein is almost restored (positive selection : $S P$ ). Similar changes in the expression of $r i$ were obtained by altering the temperature at which flies were cultured during the pupal stage. Surprisingly, a single temperature change treatment gave rise to strains in which the modified expression of $r i$ was stably inherited through more than 150 generations. There are two temperature sensitive phases within the pupal stage, one at $113 \pm 5 \mathrm{~h}$ and the other at $149 \pm 5 \mathrm{~h}$ after egg laying. The line derived from temperature change at $113 \pm 5 \mathrm{~h}$, riT113, had an $L 2$ length two times less than in the original line, $r i C$. The line derived from temperature change at $149 \pm 5 \mathrm{~h}$, riT149, had an L2 length 1.5 times greater than in riC. Genetic analysis demonstrated that expression of the $r$ phenotype was affected by polygenic modifiers. All five lines were investigated by in situ hybridisation of DNA clone of MGE (mobile genetic element) $D m-4 I 2$ to larval polytene chromosomes. A tree of similarity of MGE hybridization patterns was built by the methods of matrix clustering. The lines with the most similar expressions of the ri phenotype (riSN and riT113, riSP and riTI49, correspondingly) were found to have the most similar patterns of $D m-412$ localization and transpositions. The $D m-412$ transpositions in $S$ and $T$-lines (respectively selected and derived from temperature change), in comparison with the control line, $r i C$, were shown to be non-random by their localizations. Therefore, the similarity of patterns between the lines was mainly the result of these similar transpositions. Genetic drift and independent random changes of patterns were shown to be improbable explanations of these effects.
\end{abstract}

Key words : Drosophila melanogaster, quantitative character, temperature hereditary effects, mobile genetic elements localization patterns.

\section{Résumé}

Expression d'un caractère quantitatif (radius incompletus), effets de la température et localisation de l'élément génétique mobile $\mathrm{Dm}-412$ chez Drosophila melanogaster

La mutation radius incompletus (ri) produit une interruption dans la veine radiale $L 2$ de l'aile. Une lignée témoin ( $r i C$ ) portant la mutation $r i$ a subi une sélection divergente sur la longueur de la veine radiale. Dans la ligne riSN, la sélection négative sur ce caractère quantitatif a abouti à 
une élimination presque complète de la veine $L 2$. Dans la lignée riSP, la sélection positive a presque restauré la veine $L 2$. Des modifications similaires de l'expression de la mutation $r i$ ont été obtenues par des traitements thermiques pendant le stade pupal. De façon surprenante, un seul traitement thermique a été à l'origine de lignées dans lesquelles l'expression modifiée de la mutation $r i$ s'est transmise de façon stable pendant plus de 150 générations. Il y a deux stades de sensibilité à la température pendant la période pupale, l'un à $113 \pm 5 \mathrm{~h}$ et l'autre à $149 \pm 5 \mathrm{~h}$ après la ponte. La lignée obtenue par traitement thermique à $113 \pm 5 \mathrm{~h}$, riT113, avait une veine L2 deux fois moins longue que la lignée originelle ric. La lignée obtenue par traitement thermique à $149 \pm 5 \mathrm{~h}$, riT149, avait une veine $L 21,5$ fois plus longue que la lignée $r i C$. L'analyse génétique a montré que l'expression du phénotype $r i$ est affectée par des modificateurs de nature polygénique. Les cinq lignées ont été analysées par hybridation in situ d'un clone d'ADN de l'élément génétique mobile (EGM) Dm-412 sur les chromosomes polytènes des larves. Un arbre de similarité pour la distribution chromosomique des EGM a été construit par les méthodes de classification hiérarchique. Les lignées se ressemblant le plus quant à l'expression du phénotype $r i$ (riSN et riT113 d'une part, riSP et riT149 d'autre part) présentaient les distributions les plus semblables pour la localisation de l'élément $D m-412$. Les lignées $S$ (obtenues par sélection) et $T$ (obtenues par traitement thermique), comparées à la lignée témoin riC, présentaient des variations de la localisation des transpositions de l'élément $D m-412$ qui ne sont pas le fait du hasard. Ainsi, la ressemblance des patterns d'hybridation entre lignées s'explique principalement par des transpositions semblables. Il est improbable que la dérive génétique et des variations aléatoires des localisations de $D m-412$ puissent être à l'origine de ces observations.

Mots clés : Drosophila melanogaster, caractère quantitatif, effets héréditaires de la température, localisation des éléments génétiques mobiles.

\section{Introduction}

The investigation of structure and dynamics of mobile genetic elements (MGE) in Drosophila have opened recently new possibilities for understanding genome organization, gene expression, variability and other phenomena (see for example reviews of Rubin (1983), Ananyev (1984) and Khesin (1984)). Gvozdev, Kaidanov and colleagues (Pasyukova et al., 1984 ; Pasyukova et al., 1985 ; Gvozdev \& Kaidanov, 1986) had found a correlation between patterns of MGE localization in Drosophila chromosomes and expression of the quantitative characters viability and male sex activity in different selected lines. In this case the quantitative characteristics were the main components of biological fitness.

In this paper we describe a similar phenomenon for a different genetic system in Drosophila melanogaster involving expression of a quantitative mutant phenotype, radius incompletus (interruption of radial wing vein). Below we present a short review of our protracted investigations of some properties of this system : genetic, ontogenetic (part II) and cytogenetic (part III). The main results were derived from comparison of different Drosophila lines, developed from an original control line $(\mathrm{riC})$ by selection or temperature treatment. It will be shown that the patterns of MGE localization correlate with the expression of a quantitative character. This correlation is independent of whether these lines were derived by selection or temperature treatment. The detailed papers containing these data were published in Russian (VASILYEVA et al., $1987 a, b$; RATNER \& VASILYEVA, 1987). 


\section{The properties of investigated lines}

\section{A. The properties of control and S-lines}

The mutation radius incompletus (ri) is located at 47.0 on the third chromosome of Drosophila melanogaster (LindSLEY \& GRELL, 1968) between cytological bands $77 E$ to $78 \mathrm{C}$. The mutant phenotype consists of an interruption in the radial wing vein $(L 2)$, producing distal and proximal fragments; the remaining lenghts of wing vein giving a quantitative character which can be selected.

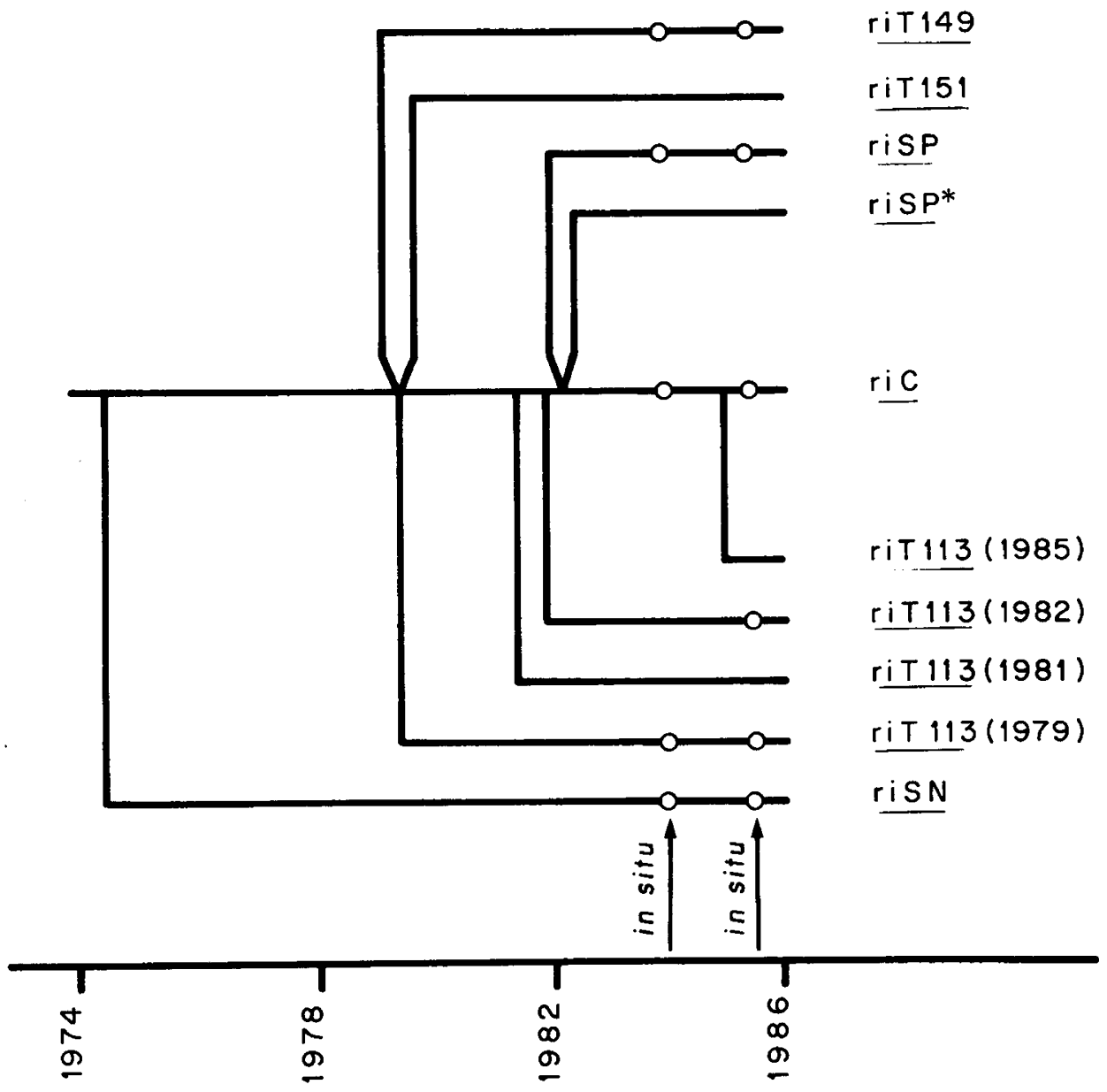

Fig. 1

A genealogical tree of the lines used. The control line, riC, is disposed at the center; below the center: the similar (by phenotype) lines riSN and riT113; above the center: the similar (by phenotype) lines riSP and riT149 (151). The arrows indicate the years when the in situ hybridization was done. 
The tree of genealogy of the investigated lines is shown in figure 1 . The original laboratory line of the $r i$ mutation was received from the Department of Genetics of Leningrad University in 1963. In 1974, flies from this line were mixed with natural populations from Novosibirsk, Uman and Batumy, to increase the genetic variability of modifier genes. From this cross, the control line, $r i C$, was derived. The $r i C$ line was cultivated for more than 300 generations at $25^{\circ} \mathrm{C}$. Forty stock bottles each containing over 100 individuals were maintained. To reduce genetic drift and inbreeding, flies from the 40 separate stocks were mixed each $30-50$ generations, and then subdivided into 40 separate stock bottles. Table 1 contains the statistical parameters of the characters in the 220th generation. Figure 2 (a) demonstrates the phenotype of the $\mathrm{riC}$ line. It is reasonably stable (see fig. 3 and 5 , curves $2, \mathrm{~A}$ and $\mathrm{B}$ ). We took this line as a control for temperature treatment and selection.

TABLE 1

The average length of proximal $\left(\overline{\mathrm{X}}_{1}\right)$ and distal $\left(\overline{\mathrm{X}}_{2}\right)$ fragments of female wing radial vein from the different lines of Drosophila melanogaster

\begin{tabular}{l|c|c|c|c}
\hline \hline Line & $\begin{array}{c}\text { Year of line } \\
\text { foundation }\end{array}$ & Generation & $\bar{X}_{l} \pm s$ & $\bar{X}_{2} \pm s$ \\
\hline$r i C$ & 1974 & 220 & $1.91 \pm 0.05$ & $0.39 \pm 0.07$ \\
$r i S N$ & 1974 & 220 & $0.50 \pm 0.02$ & absent \\
$r i T 113$ & 1979 & 140 & $1.05 \pm 0.05$ & absent \\
$r i T 149$ & 1979 & 140 & $2.45 \pm 0.09$ & $1.47 \pm 0.08$ \\
$r i S P$ & 1982 & 40 & $3.80 \pm 0.02$ & $2.20 \pm 0.03$ \\
\hline
\end{tabular}

Similar data were found for males.

In 1974, a sub-line of $\mathrm{riC}$ was taken for mass selection for elimination of the $\mathrm{L2}$ vein (negative selection). This was achieved within 70 generations and the selected line, riSN, was established (VASIlyeva \& Nikoro, 1976 ; Vasilyeva, 1979, 1984 a). The dynamics of selection is depicted in figure 3 (curves $3, \mathrm{~A}$ and B). The typical phenotype of riSN individuals is shown in figure 2 (b) ; table 1 contains statistical parameters of the riSN line in the 220th generation. This line is cultivated at $25^{\circ} \mathrm{C}$ under identical conditions to $r i C$. After cessation of selection, the line riSN had not returned to its starting state, but had inherited the selected phenotype during more than 150 generations. In other words, this line came to a stable state of new genetic homeostasis (VASILYEVA, 1984 a). A distal fragment of a vein was lost irreversibly.

In 1982 a new sub-line was taken from riC. Several separate replicates were mass selected for restoration of the $L 2$ vein (positive selection). This was achieved within 30 generations with random crossing within replicates. From this the line riSP was established. The dynamics of selection response is depicted in figure 3 (curves $1, \mathrm{~A}$ and B), where the arrows indicate the generation of total restoration of the radial vein. A typical phenotype of invididuals is shown in figure 2 (c) ; table 1 contains statistical parameters of a line in the 40th generation. This line was cultivated at $25^{\circ} \mathrm{C}$ under identical conditions to those used for $r i C$. After selection was stopped, the line riSP also did not return to the starting state.

An analysis of response of these lines to selection led us to distinguish at least three groups of modifier genes, affecting the expression of the ri mutation : 1) those of the distal fragment ; 2) those of the proximal one ; 3) pleiotropic modifiers with common effects (VasilyeVA \& NiKoro, 1976 ; VasilyeVa, 1981, $1984 b$ ). 

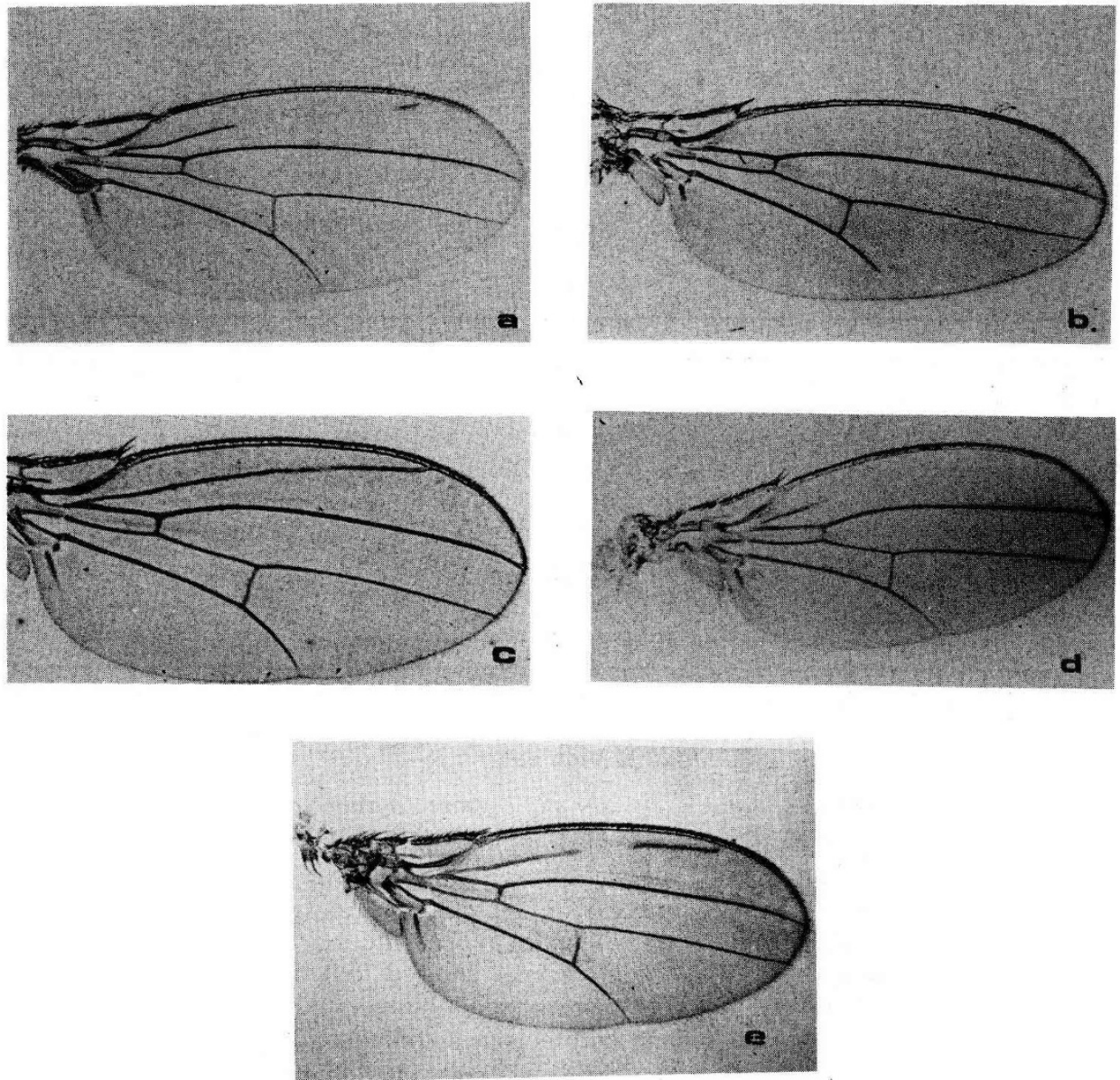

Fig. 2

The fly wings from different lines of Drosophila melanogaster : a) starting and control form (riC) ; b) the results of selection for decreasing of $\mathrm{L} 2$ (riSN), distal fragment is totally eliminated;

c) the result of selection for increasing of $\mathrm{L} 2$ (riSP), the radial vein is totally restored;

d) the result of single step-wise temperature treatment $\left(29^{\prime \prime} \rightarrow 18^{\circ} \mathrm{C}\right)$ of the line riC at the stage of $113 \pm 5 \mathrm{~h}$ after egg laying, the subsequent generations being cultivated at $25^{\circ} \mathrm{C}$ (riT113);

$e)$ the result of the same treatment at the stage of $149 \pm 5 \mathrm{~h}$ after egg laying, the subsequent generations being cultivated at $25^{\circ} \mathrm{C}$ (riT149). 


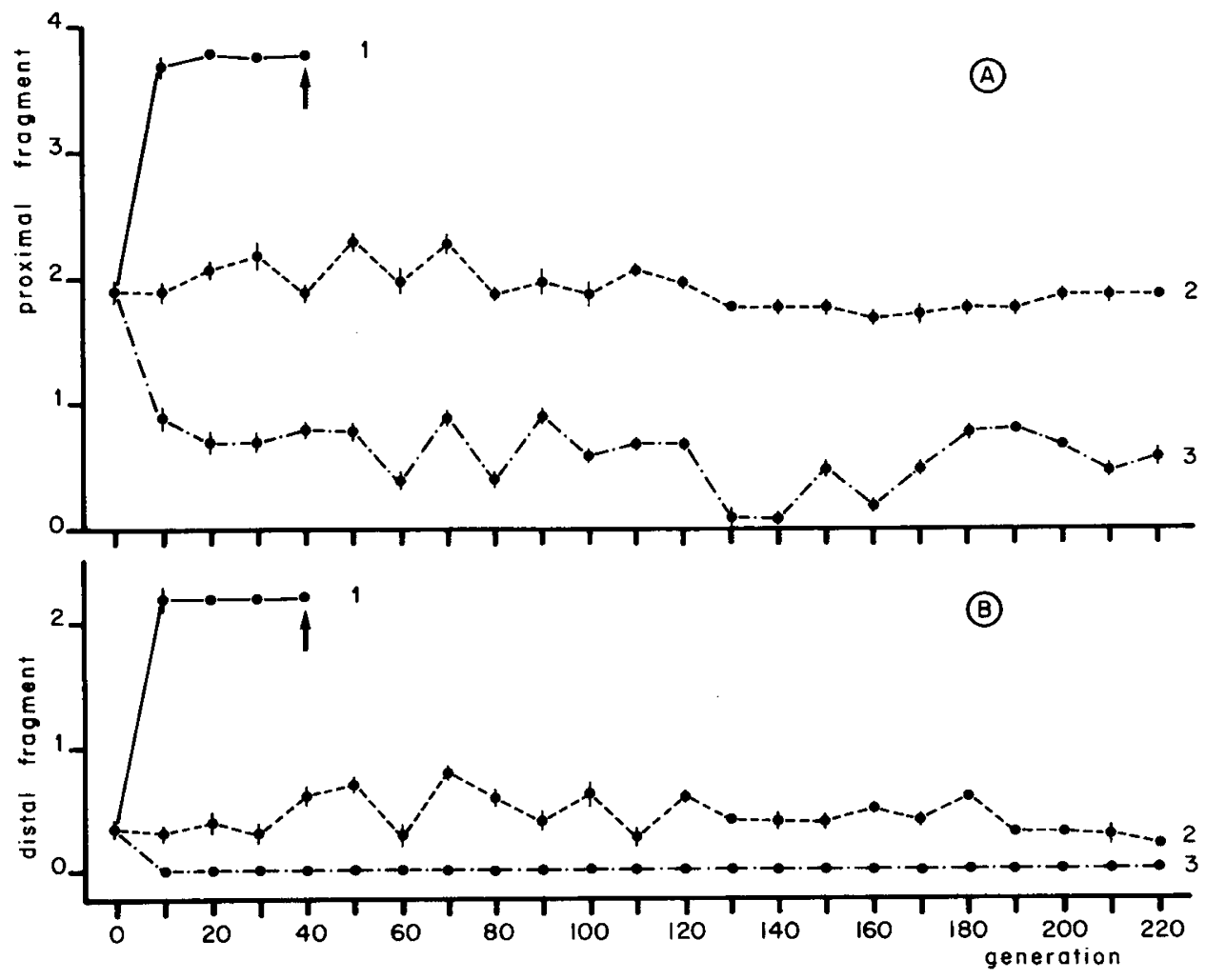

FIG. 3

The dynamics of response to selection in positive and negative directions of female wing radial vein fragments - proximal $(A)$ and distal $(B)-$ in lines riSN and riSP. Designations $: 1=\mathrm{riSP}, 2=\mathrm{riC}$, $3=$ riSN. The arrow indicates the generation where two fragments join one another.

A genetic analysis of this system, made by substitution of chromosomes from $S$-lines with marked ones from the control line, showed that all three large chromosomes contribute additively to the expression (VAsilyeva, $1984 b$; Vasilyeva \& Mukhina, 1985). The first and second chromosomes contain, at the least, nine regions with modifiers located : seven for the proximal fragment and two for the distal one.

Thus, based on the comparison of the results of genetic analysis of $S$-lines and the control line, $r i C$, we conclude that the genetic system of $r i$ expression corresponds well with the classical polygene model (MATHER \& JINKS, 1982) : expression of the oligogene mutation $r i$ is modified by a group of modifier genes with small effects and with different locations.

\section{B. The properties of $\mathrm{T}$-lines}

In 1979 we began the investigation of temperature influence on the expression of genetic system ri (VASIlleva, 1982, $1984 \mathrm{c}$ ). Some different culture regimes were used. The most clear-cut results were obtained under the next scheme of temperature experiment. 
Fertilized females from all replicates of the control line $\mathrm{riC}$ were carefully mixed and then distributed among 500 tubes at 30 females per tube. Eggs were laid within an hour at $25^{\circ} \mathrm{C}$, after which females were removed and tubes were cultivated at $29^{\circ} \mathrm{C}$. After this, each hour three tubes were transferred to $18{ }^{\circ} \mathrm{C}$ and were cultured at this temperature until the imago stage. The age of individuals was counted from the hour of egg laying. The generation treated by temperature was considered as $F_{t}$. All the subsequent generations were cultivated at $25^{\circ} \mathrm{C}$ under identical conditions to those of $r i C$.

The entire pupal stage (up to imago) was found to be sensitive to temperature treatment, as analysed by expression of the $r i$ character in the $F_{v}$. Most of these changes, however, were nonhereditary (VAsilyeva, $1984 b, c$ ). However, there are two narrow sensitive periods within the pupal stage, when the change of culture temperature can result in hereditary changes of phenotype (fig. 4).

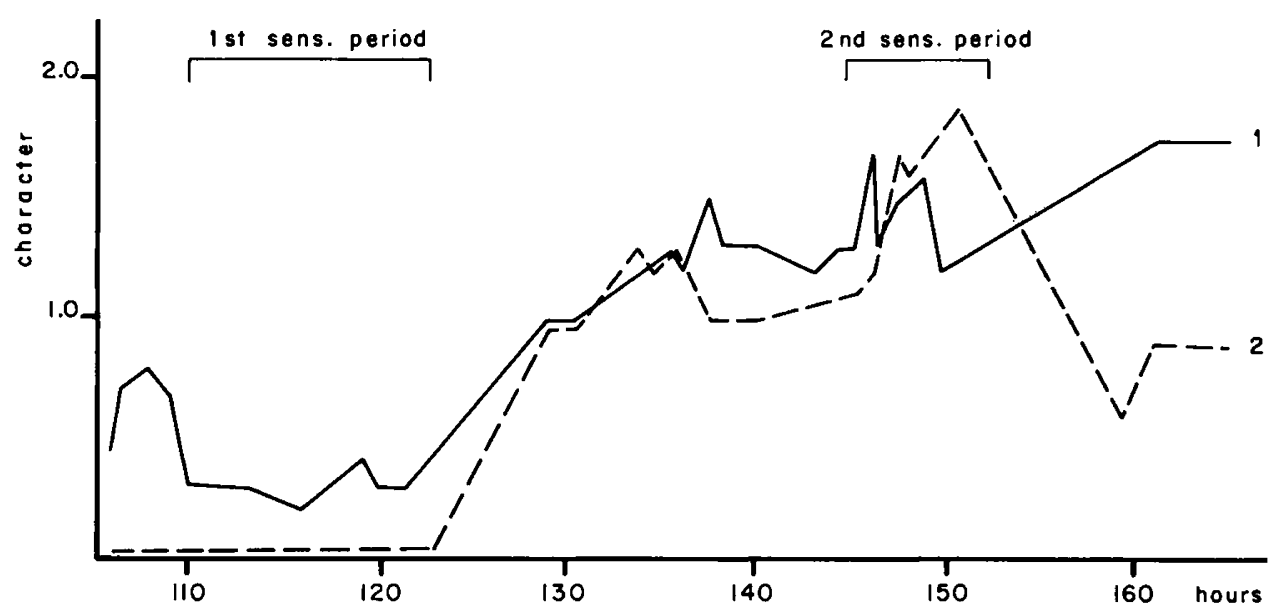

FIG. 4

A dependence of an average length proximal (1) and distal (2) fragments of wing radial vein from a stage of a culture riC, when was a temperature treatment. The two temperature-sentitive periods are indicated.

The first of them was found at $113 \pm 5 \mathrm{~h}$ at $29^{\circ} \mathrm{C}$, corresponding with the yellow pre-pupal stage (VASILYEVA, $1984 b, c$ ). Temperature treatment at this stage resulted in reduction of both distal and proximal fragments in $F_{I}$ and the next generations. Differences were found in tubes treated at different times within the sensitive periods. Tubes treated simultaneously contained flies with very similar phenotypes. For subsequent breeding we took only tubes where all flies had completely lost distal fragment of L2. In following generations, partial restoration of a proximal fragment occurred. The phenotype stabilized at the level of two times less than in $r i C$. This «temperature " line ( $T$-line) was named riT113. The dynamics of the vein-fragment lengths in the following generations is depicted in figure 5 (curves $3, \mathrm{~A}$ and B). Figure 2 (d) demonstrates a typical phenotype, and table 1 contains the statistical parameters of a stabilized line at the 140 th generation. 

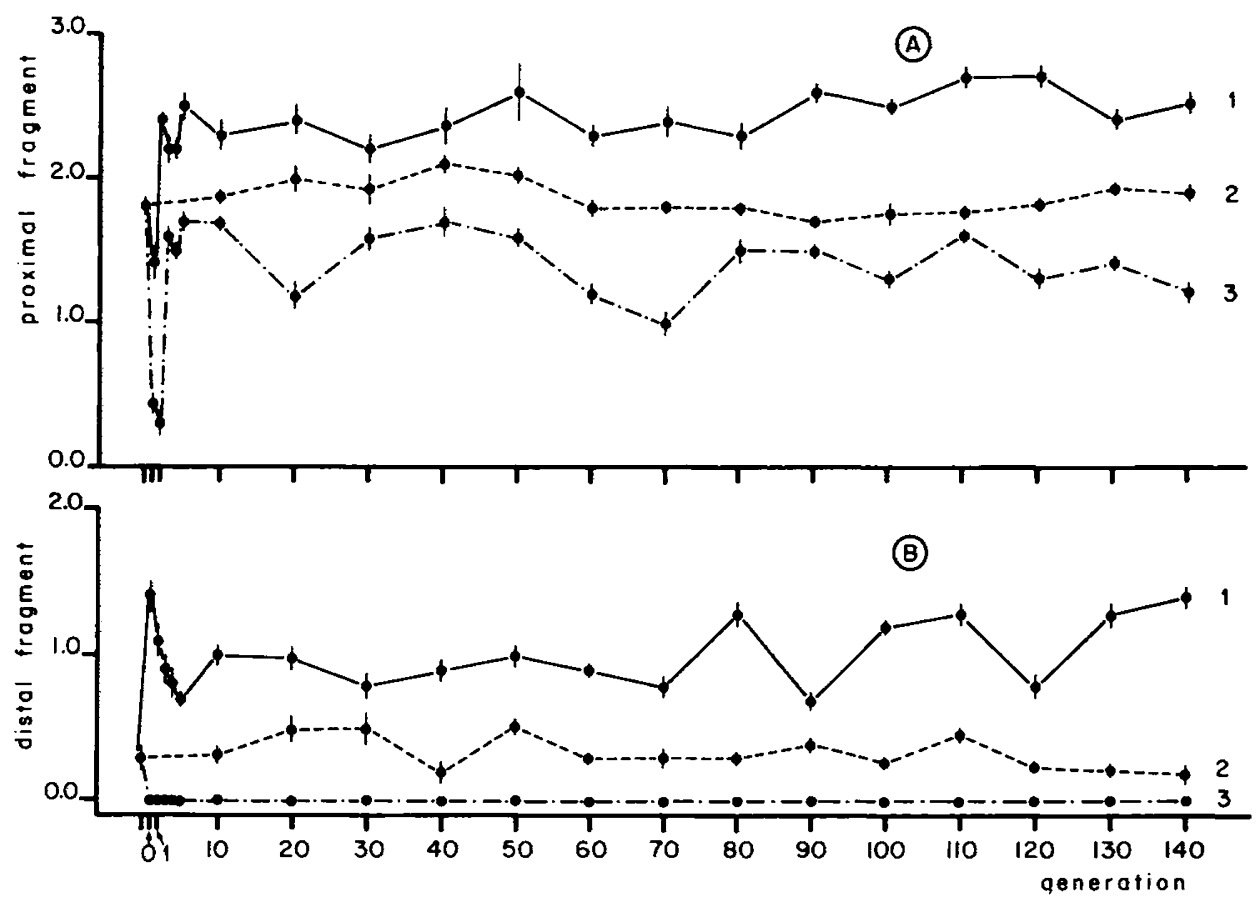

Fig. 5

The dynamics of expression stabilization of proximal $(A)$ and distal $(B)$ fragments of female wing radial vein in subsequent generations $\left(\right.$ at $\left.25^{\circ} \mathrm{C}\right)$ after the temperature treatment $\left(29^{\circ} \mathrm{C} \rightarrow 18^{\circ} \mathrm{C}\right)$ in $\mathrm{F}_{0}$. Designation : $I=\mathrm{riT} 149,2=\mathrm{riC}, 3=\mathrm{riT} 113$.

The second temperature-sensitive period was found at $149 \pm 5 \mathrm{~h}$ at $29^{\circ} \mathrm{C}$, corresponding to the late (dark) pupal stage (VAsilyeva, $1984 \mathrm{~b}, \mathrm{c}$ ). The temperature change at this stage results within the $F_{0}$ generation in a noticeable reduction in the length of the proximal fragment and an appreciable growth of the distal one. After generations $F_{1}-F_{2}$ the character was stabilized at about 1.5 times greater than in $r i C$. This $T$-line was named riT149. The dynamics of vein-fragment lengths in the following generations are depicted in figure 5 (curves $1, A$ and B). Figure 2 (e) demonstrates a typical fly phenotype, and table 1 contains the statistical parameters of the stabilized line at the 140th generation.

The most surprising property of both $T$-lines is the induction, by a single temperature-stress treatment, of a changed phenotype which is stably inherited through more than 150 generations at $25^{\circ} \mathrm{C}$ (see fig. 5, curves 1 and $3, \mathrm{~A}$ and $\mathrm{B}$ ).

However, some properties of temperature experiments should be noted. Firstly, among the many cultures of $r i C$, treated by temperature change at different stages within the temperature-sensitive periods, not all cultures expressed the stably inherited altered phenotype. This might result from incomplete synchronisation of development. However, after the temperature treatment during the first sensitive period, the changes in $F$, were always towards decreased, and during the second one towards increased 
values of a character, i.e. were opposite. Therefore, to derive the most contrasting lines, we took cultures with the most extreme phenotypic changes and with the most stable inheritance. Moreover, for the minimalization of ontogenetic scattering only imago flies were taken emerging during the initial 4-5 hours from all cultures.

\section{A preliminary interpretation of genetic data and temperature effects}

First of all, we can conclude on the basis of the results of genetic analysis of control (riC) and two $S$-lines ( $r i S N$ and $r i S P$ ), that the genetic system of expression of $r i$ is a typical polygenic system (see MATHER \& JinKs, 1982). This means that, apart from oligogenes ( $r i$ and, perhaps, some others), this system contains numerous modifier genes, affecting the expression of the $r i$ phenotype.

The hereditary changes resulting from change in culture temperature might not be similar to the effect in the $F_{n}$ (see, for example, fig. 5 , curve $1, \mathrm{~A}$ ). Hence, it seems possible, that hereditary events occur within modifier genes in the germ-line. These events are of mass scale, and cannot be ordinary mutations. The fact that temperature treatments at two different sensitive periods result in changes in the hereditary character in opposite directions implies that there is a change in the state of germ-line chromosomes between the two periods. A dependence of the character expression on the time of temperature treatment during the sensitive periods could be explained by non-synchronisation of individual development.

It is necessary to discuss the possible role of selection in the observed temperature effects. The control line $r i C$ is heterogeneous for modifier genes. It seems possible that temperature treatment is either a direct selection factor, or the provocative background, within which different polygene genotypes could express their different fitness. This selection could affect either individuals in the $F_{\theta}$, or the developing germ-line cells of these individuals at the pupal stage.

None of these hypotheses, however, seem likely. If the mass change of phenotypes in $F_{l}$ resulted from very strong selection at the sensitive periods in the pupal stage, then a high incidence of pupal mortality would be expected. This was not observed. As regards selection of germ-line cells at the sensitive periods, modifier genes of the radial vein are then nonfunctional, and so their combinations could not be estimated by selection either of this character, or of indirect expression.

The selections of $T$-lines similarly could not, in itself, cause quick and mass hereditary shifts of the average population phenotypes, as whole cultures, rather than individual flies, with the extreme expression of the characters, were bred from. The degree of expression in $F_{l}$ depends on the time of temperature treatment within sensitive periods of $F_{0,}$. Therefore, the role of temperature treatment could be only in inducing heritable changes. Thus temperature effects during the sensitive periods probably do not select for preexisting genotypes.

It is important to underline that very similar temperature effects, though genetically unexplored, were discovered earlier by Svetlov \& Korsakova $(1966,1972)$ in Drosophila. They found that expression of the oligogene mutations forked and eyeless was dependent on duration of heating or cooling of Drosophila females (i.e. actually, of their maturing eggs), and was further inherited for tens of generations without any additional treatment. 
Recently among the factors capable of influencing the expression of quantitative characters, increasing attention has been paid to mobile genetic elements (MGE) (Georgiev \& Gvozdev, 1980 ; Pasyukova et al., 1984, 1985 ; Gvozdev \& Kaidanov, 1986). Some hypotheses have been proposed about the possible role of MGE in such phenomena (Vasilyeva \& Zabanov, 1984/1985 ; Gvozdev \& Kaidanov, 1986 ; VasiLYEVA et al., 1985, $1987 a, b$; RATner \& VAsilyeVA, 1987). Below we present our data in favour of this viewpoint.

\section{A chromosome localization of MGE $D m-412$}

The described $S$ - and $T$-lines, derived by us, represent unique material for investigation of different aspects (genetic, selectional, ontogenetic, cytogenetic and so on) of MGE influence on the expression of quantitative character in Drosophila.

\section{A. Materials and methods}

\section{The Drosophila lines}

Drosophila melanogaster lines $r i C$, riSN, riSP, riT113, and riT149 were used as described above.

\section{DNA extraction and hybridization in situ}

The extraction of plasmid DNA, containing the MGE Dm-412 (mdg-2) was made by the alkaline method (BIRnBoim \& Doly, 1979). DNA was labelled by nicktranslation (MANIATIS et al., 1982). For hybridisation, DNA preparations with specific activity of $2-6 \cdot 10^{6} \mathrm{cpm} / \mu \mathrm{g}$ were used. Hybridization was done in $4 \times S S C$ and $10 \%$ dextransulfate. Hybridization in situ followed the method of Gall \& Pardue (1971).

\section{A method of building the similarity tree}

Based on the data of hybridization in situ, the joint table was constructed (table 2), containing the patterns of MGE $D m-412$ localization along the segments of cytological map of Drosophila melanogaster polytene chromosomes. Designations of segments correspond to the map of Bridges (see Lindsley \& Grell, 1968).

The columns of the table correspond to different individuals (table 2). For each pair of columns the sum of differences of MGE sites was counted. This values (a distance between the columns) is the quantitative measure of the difference of two patterns of MGE localization. These distances for all the pairs of columns (individuals) were collected into the joint matrix of distances $\left(\mathbf{D}_{i j}\right)$. Based on this matrix, the tree of similarity of flies and lines was built by conventional clustering methods (the unweighted pair-group method using arithmetic averages, UGPMA : see SNEATH \& SoKAL, 1973).

A procedure of similarity tree building consists of two parts : a building of the tree topology, and an estimation of the tree branch lengths. A tree topology (i.e. a number and sequence of dichotomic branching points) was built by the following method 

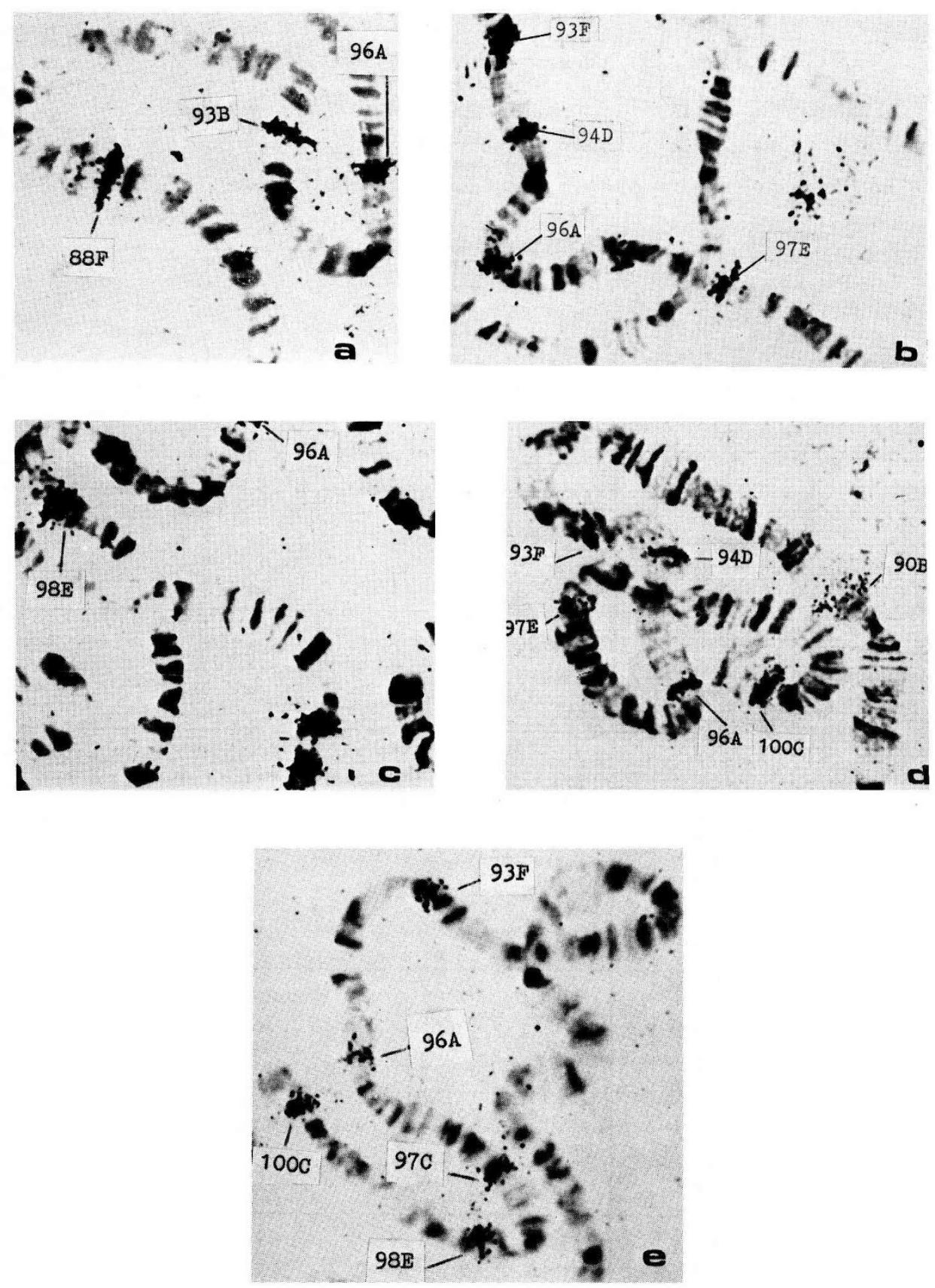

Fig. 6

The examples of MGE Dm-412 hybridization with segments of $\mathrm{R}$-arms of polytene third chromosomes of Drosophila melanogaster salivary glands in situ for five lines: a) $\mathrm{riC}$, b) riSN, c) riSP, d) riT113, e) riT149. 


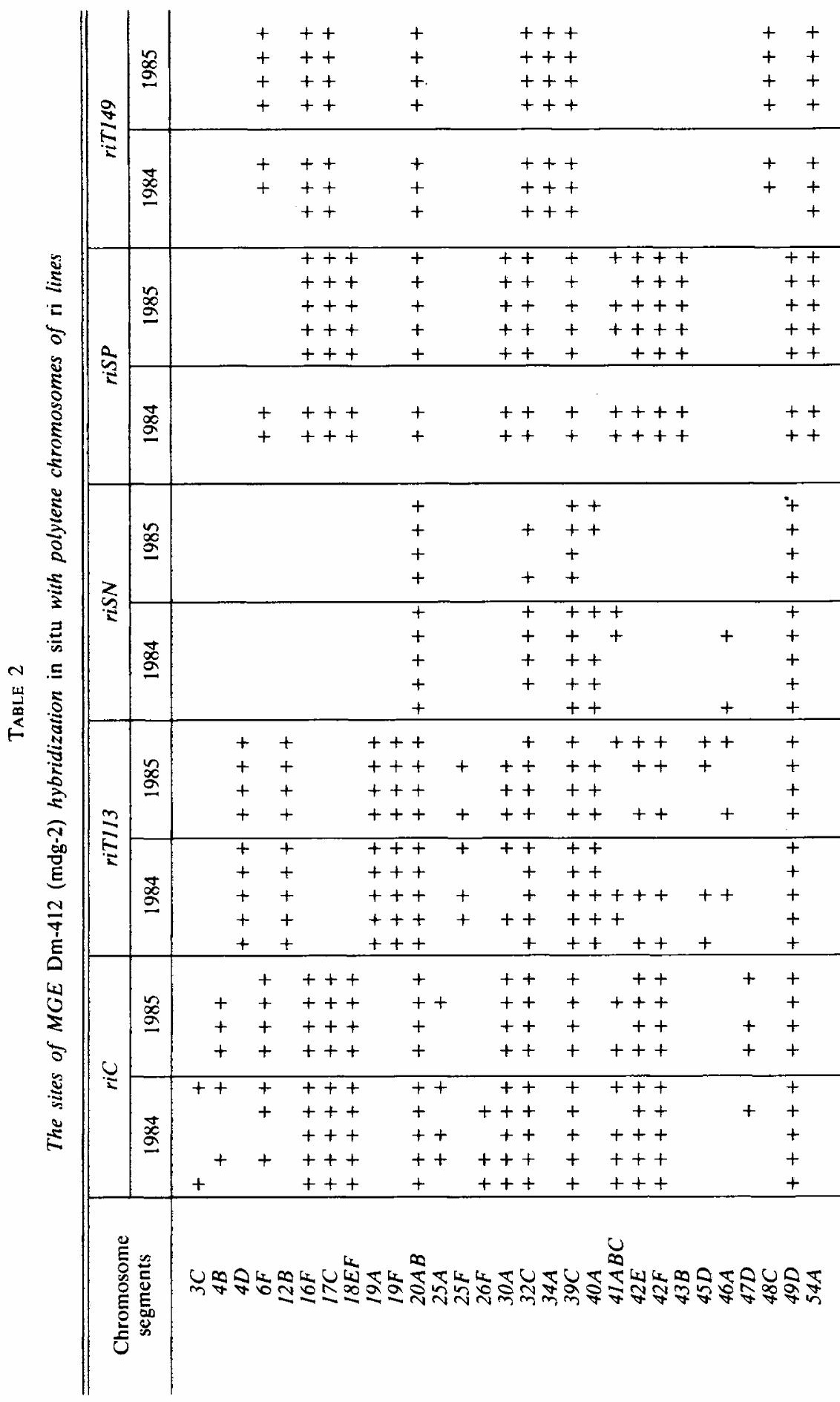


(UPGMA : see SNEATH \& SoKal, 1973). From the distance matrix $\mathbf{D}_{i j}$ was extracted the minimal element, a pair of the most similar individuals, corresponding to a pair of exterior points of the tree. This pair was substituted by one point with the average distance from the all other exterior points. In other words, a low level dichotomic branching pattern was built. This procedure was reiterated until all the exterior points were connected into a joint tree.

Then, based on a matrix of direct distances $\mathbf{D}_{i j}$, and using the $\chi^{2}$ method, the set of the branch lengths $\{L\}$ for a built tree was calculated. As a result, the calculated branch-lengths give a measure of average distinction numbers between corresponding branch points of a tree. Though the values of $D_{i j}$ are always integers, the average branch-lengths can be fractional (fig. 7).

It is important to note that although all five lines were derived from the initial line $\mathrm{riC}$, the tree of similarity (fig. 7) does not reflect the consequences of divergence (fig. 1). Instead only distinctions of MGE localization patterns, arising either by selection or by temperature treatment, are represented. Thus, this tree represents only the results of classification. The branch points correspond not to the ancestral forms, but are only the results of clustering by similarity. For this reason, a search for the tree root would be uninformative.

\section{Calculation of correlation coefficient}

A calculation of correlation coefficient for alternative characters was achieved by standard methods (see, for example, URBAKH, 1964).

\section{B. The results of hybridization in situ and estimation of similarity of MGE localization patterns}

Figure 6 depicts some examples of MGE Dm-412 hybridization in situ with polytene chromosomes of larval salivary glands from all the five lines : riC, riSN, riSP, riT113, riT149. Table 2 contains the joint results of this hybridization.

In accordance with the tree building procedure (see III.A.3), each pair $(i, j)$ of the columns was characterised by their distance $D_{i j}$ (by number of distinct symbols $(+$ or gap) in homologous segments). As a whole, these values composed the matrix of distances, which is very bulky and not presented here. The corresponding tree of similarity of individuals and their groups, built on the basis of this matrix, is shown in figure 7 .

The points of the tree (the external points) correspond to real individuals and to columns of table 2. Clearly, the sums of lengths of the routes between the individuals from different lines are very different. Thus, riSN and riT113 have route length values of 16-17 ; riSP and riT149, of 14 ; but riSN and riSP have differences of 32-33, and $r i S N$ and $r i C$, differences of 33-34. At the same time, the routes within $T$-line riT113 are much less (from 5 to 13 ), as are routes within the other lines.

First of all, as expected, the individuals form one line always had more similar patterns of MGE $D m-412$ localization, than from different lines. The corresponding external points are connected by the shortest routes. In each line the sites of MGE binding can be subdivided into the stable and variable (heterogeneous) ones within the sample. Since the samples are not large (7-9 individuals), it is clear that variability is 
reliably determined, but stability only to $12-14 \%$. Distinctions between the individuals of one line result only from variable positions. These correspond to sub-branches of the tree structure (fig. 7).

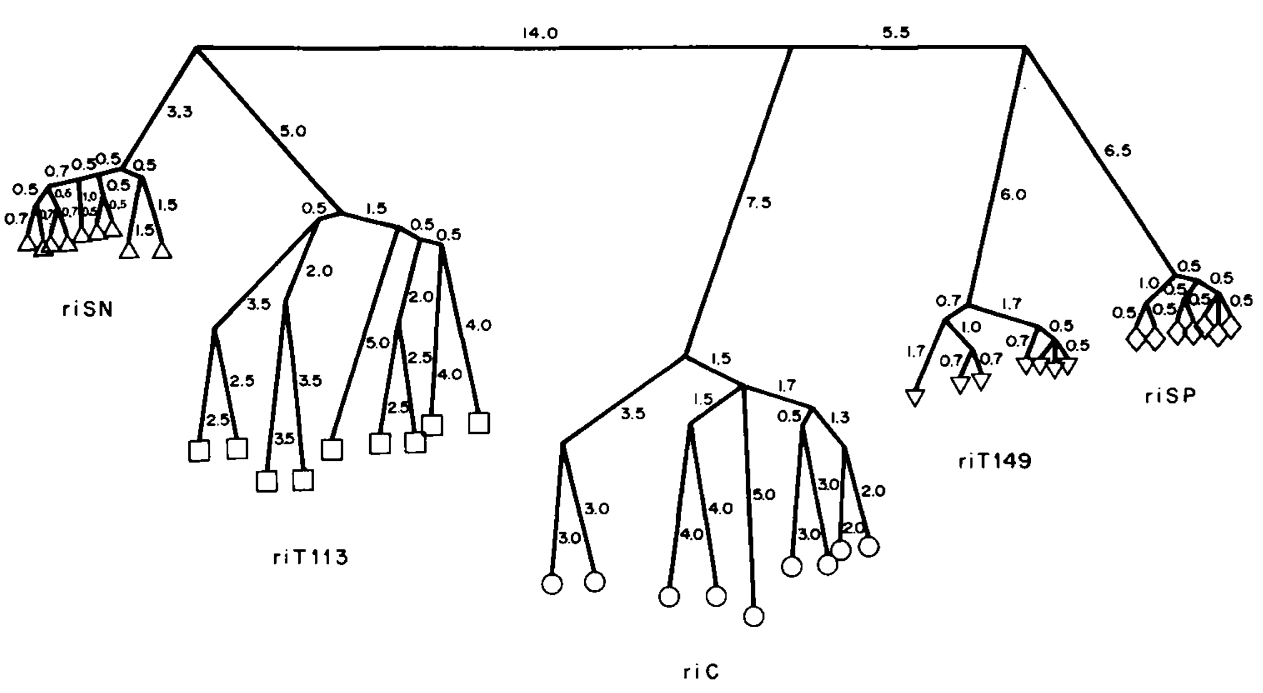

FIG. 7

The tree of similarity of patterns of $M G E$ Dm-412 localization for five lines, developed from initial riC. Designations : $O=$ riC, $\triangle=\operatorname{riSN}, \diamond=\operatorname{riSP}, \square=$ riT113, $\nabla=$ riT149. This tree was built basing on a matrix of distances by the unweighted group pairs method (SNEATH \& SoKAL, 1973) (see section III.A.3.) in accordance with the data of table 2.

The most striking result is that the lines with the most similar quantitative character expressions (riSN and riT113, riSP and riT149, correspondingly, see fig. 2 and table 1) are also the most similar by the patterns of MGE Dm-412 localization (see fig. 7). For the lines riSN and riT113 the phenotypic resemblance, first of all, suggests that the distal fragment of $L 2$ is totally lost (fig. 2 and table 1). It is important to emphasize that the lines, similar by phenotypes, were developed in totally different ways either by selection over many generations, or by a single temperature treatment without any selection (see part II). Nevertheless, the phenotypic resemblance was accompanied by similarity of MGE $D m-412$ localization patterns.

All four descendant lines were developed at different times from $r i C$, which continues to be heterogeneous (table 2). It would be expected that different lines diverged by different amounts as a result of processes such as genetic drift, and had different positions in the tree of similarity (fig. 7). However, that is not the case. The $S$ - and $T$-lines not only had lost some sites of hybridization, but had acquired many new ones in comparison with $\mathrm{riC}$. The control line $\mathrm{riC}$ has approximately the same distance from all the four descendant lines.

The recovered similarity is independent of the method of clustering used for analysis of experimental data. Moreover, the calculation of correlation coefficients of alternative characters for all the line pairs (table 3 ) results in highly reliable values particularly for those pairs, which were the most similar by the tree (fig. 7). 
TABLE 3

The correlation coefficients for alternative characters between the patterns of MGE Dm-412 localization in chromosomes of larvae from different lines

\begin{tabular}{c|c|c|c|c}
\hline \hline riT113 & riC & riT149 & riSP & \\
\hline$+0.60^{* * *}$ & +0.01 & +0.14 & -0.09 & riSN \\
& +0.01 & -0.23 & -0.08 & riT113 \\
& & +0.03 & +0.09 & riC \\
& & & $+0.49^{* * *}$ & riT149 \\
\hline
\end{tabular}

${ }^{* * * *}$ : values significant with $P<0.001$. The other correlations are not significant.

For every line the hybridizations in situ were done twice : in 1984 and in 1985 with a time interval greater than 30 generations (fig. 1). Table 2 contains the corresponding parts of the samples. A statistical comparison of the distributions corresponding to these groups of individuals, by the $\chi^{2}$ method shows that significant differences between such subgroups within all five lines are absent (table 2). Thus the patterns of MGE localization are stably maintained within the lines.

Besides, in 1985-1986 we obtained additional data for small samples of $T$-lines riT113, independently maintained since 1982 and 1985 (fig. 1). The significant differences between samples from the riT113 lines of 1979,1982 and 1985 were absent. At the same time, all five lines, represented in figure 7 , show significant differences. Hence, these preliminary data indicate stable reproducibility (by statistical criteria) of MGE localization patterns after the expected maintainance of $T$-line riT113 over several years.

\section{Discussion of the results}

Firstly, let us make sure that the MGE localization patterns cannot be explained by random genetic drift in a population of finite size. On this hypothesis, the initial line $r i C$ is heterogeneous and polymorphic at many sites of chromosome MGE localization, and the descendant lines have lost part of this variability by genetic drift. However, all lines were cultivated in 20-40 replicate cultures, each containing more than 100 individuals. The replicates were maintained independently for 30-50 generations; then they were mixed and again were randomiy subdivided into 40 new replicates. If the effective replicate size $N_{e}=N>100$, then the average time for the random fixation of one binding site is :

$$
I_{t}(p)=\frac{4 N_{e}(1-p)}{p} \ln (1-p),
$$

and for the random loss of one binding site :

$$
t_{2}(p)=\frac{N_{e} p}{(1-\mathrm{p})} \ln (p),
$$


where $p$ is initial frequency of the binding site in the population (CRow \& KImURA, 1970). If $p=0.5$, both formulae become identical, and give the average time of fixation (or loss) of the site as $t(p)=4 N_{e} \ln (0.5)=2.8 N_{e}>280$ generations in our case.

Hence, after 30-50 generations of isolated cultivation of replicates a random fixation or loss of MGE localization sites could not became the dominating event. After every cycle of crosses of replicates this process begins again. Moreover, in the course of genetic drift (without regards to MGE transpositions : see below) there are expected only site losses, whereas a substantial number of differences between samples of the lines were the acquisitions of novel sites of MGE hybridization in comparison with riC (see below). Finally, the distances between lines within a tree in this case tend to increase in successive generations. Such an increase was not observed (see below).

The other stochastic hypothesis suggests that the sites of MGE localization change because of sufficiently frequent random transpositions, connected neither with selection, nor with temperature treatment. In this case the lines would be polymorphic, the average distances between them being larger if they diverged earlier (fig. 1). Comparison of the lines, branching of $\mathrm{riC}$ in different years, shows that this is not the case. Two $T$-lines, riT113 and riT149, cultivated for more than 140 generations, have different distances from the $S$-line $r i S N$ (fig. 7) (the same case, from riSP). The lines riSN and riT113, branched off more than 220 generations ago, have the shortest distance between each other on the tree. Finally, all the four descendant lines have approximately the same distances for $\mathrm{riC}$, though they were initiated non-simultaneously (fig. 1). Thus, a tree of similarity (fig. 7) is totally unlike the genealogical tree of these lines (fig. 1). In other words, the distances between lines are independent of gradual, «background " accumulation of transpositions.

Let us consider in detail the comparative changes of MGE localization patterns in relation to $\mathrm{riC}$ (table 4 ). Firstly, all the four lines, branched off $\mathrm{riC}$ in different years, had lost 10 common sites (comparing with $\mathrm{riC}$ ), all of them being heterogeneous in $\mathrm{riC}$ (see table 4, column 1). No common novel sites are present. The lost sites are distributed more or less evenly along the chromosomes.

This interesting fact could be interpreted in two extreme ways : 1) either all 10 novel sites were fixed in the control line $r i C ; 2$ ) or all the 10 losses were fixed in the other four lines. Above we had excluded the possibility of the phenomenon resulting from drift or from accumulation of random transpositions independent of selection and temperature treatment. Hence, the most probable are the variants of non-random, canalized phenomena. In the first case all the 10 novel sites could arise only in the last stages of cultivation of $r i C$, after divergence of riSP $(\sim 40$ generations up to hybridization in situ, see fig. 1). Moreover, after this time two different steps of hybridization in situ within the interval of 30 generations had not revealed the significant distinctions between two samples (by patterns of MGE localization, see table 2). Finally, in the sample from a still more recently derived line, riT113 (of 1985 initiation), not included in table 2 , the 10 previously observed losses were found again (in comparison with $\mathrm{riC}$ ). Thus, all 10 losses were fixed in the $r i C$ line during only 10-15 generations. Such an event seems to be very improbable in a line untreated by any special factor.

In the second case, all the 10 common differences from $\mathrm{riC}$ could be the result of common canalized losses in the four descendant lines. This implies that these sites are characteristic for the phenotype of $r i C$, heterogeneous for MGE sites within the $r i C$ line, and quickly lost after active narrowing of variability of phenotypic expression. The most likely hypothesis is that spontaneous MGE transpositions are rare, but obviously 


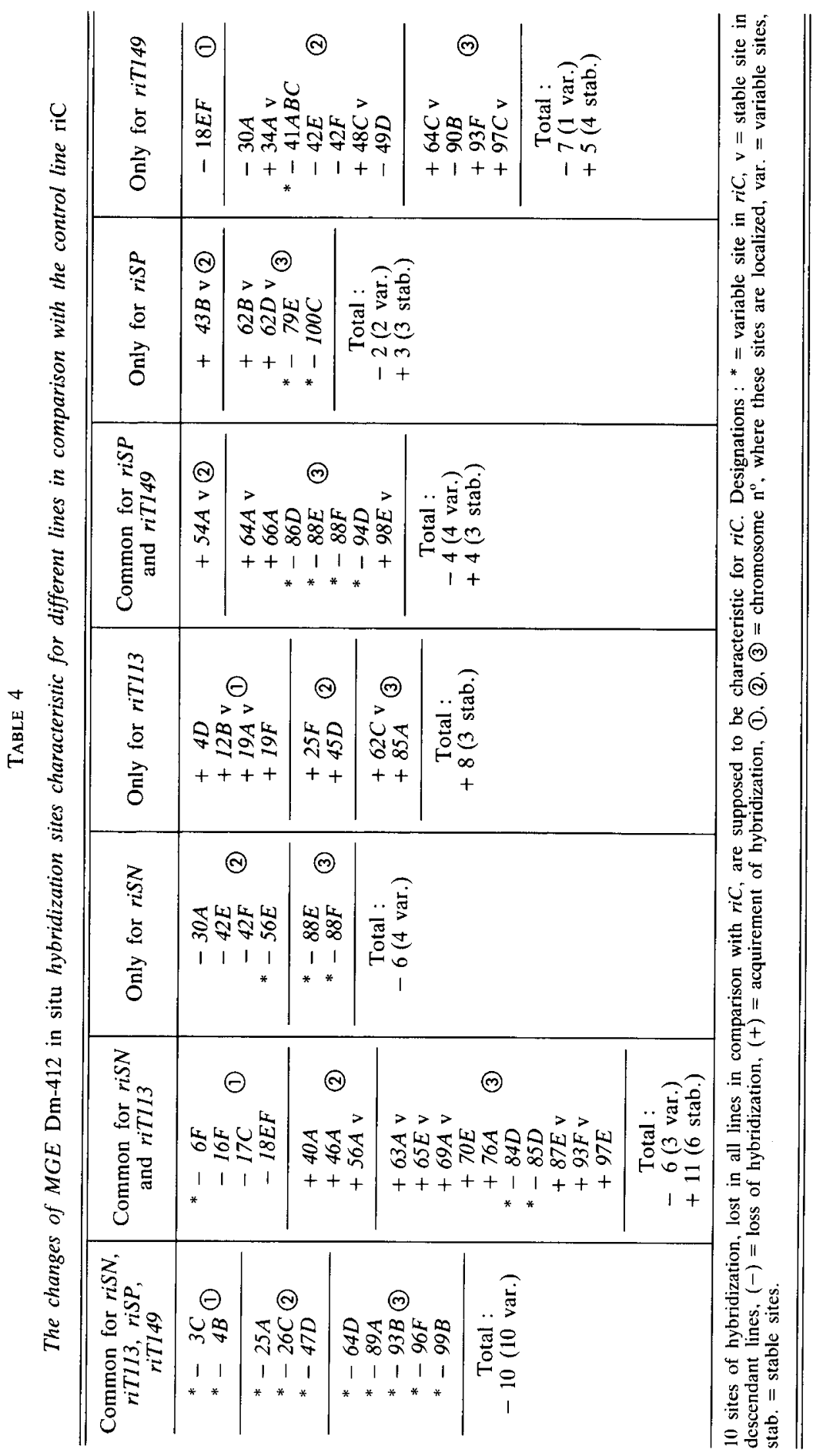


non-random by their positions, and could be stimulated by temperature treatment or favoured by selection.

Other facts agree with this hypothesis. It is obvious that the important part of pattern similarity between the lines derived from two sources : a) partially from the conservation of common sites, originated from the initial control line $r i C(\sim 50 \%)$; b) partially from new common alterations of the initial pattern of the $r i C$ line, arisen from non-random canalized transpositions. So, the similarity between the phenotypically similar lines riSN and riT113 is provided by 15 common sites, originated from riC (see table 2), 10 common site losses (in comparison with $\mathrm{riC}$ ) for all the descendant lines (see table 4, column 1), 6 losses and 11 novel sites, common for this pair of the lines only (see table 4, column 2), i.e. 27 common alterations among 33-35 changed positions. In addition the riSN line lost 6 specific sites (see table 4, column 3) and the riT113 line acquired 8 novel sites (see table 4, column 4). These effects were most obvious in the X-chromosome : riT113 lost 6 sites and acquired 4 novel ones, and only a single site is constant ; riSN lost the same 6 sites, the novel sites are absent, and the same single site is present (table 2).

The other pair of phenotypically similar lines - riSP and riT149 - had very similar properties : 14 common sites, originated from $\mathrm{riC}$ (table 2), 10 site losses, common for all descendant lines (see table 4, column 1), 4 losses of variable sites and 4 novel sites ( 3 being stable), common for this pair of lines (see table 4 , column 5), i.e. 18 common alterations among 23-30 changed positions. Moreover, riSP lost 2 variable specific sites and acquired 3 novel stable sites (see table 4, column 6) ; and riT149 lost 7 specific sites ( 1 being variable) and acquired 5 novel ones ( 4 being stable) (see table 4 , column 7). It is remarkable that the sites, in which changes contributed to the similarity of the line pairs riSN and riT143, riSP and riT149, are located mainly in the chromosome, which contains also the oligogene ri (see table 4, columns 2 and 5).

Thus, the spectra of changes of MGE localization in the lines with similar phenotypes are obviously non-random, and the similarity of patterns is mainly the result of these changes (see also VAsilyeva \& Zabanov, 1984/1985 ; Vasilyeva et al., 1985).

The similarity of MGE localization patterns between Drosophila lines, selected for similar expression of a quantitative character, has already been indicated (GeORGIEv \& Gvozdev, 1980 ; Gvozdev et al., 1981 ; Pasyukova et al., 1984 ; Gvozdev \& KaidaNov, 1986). The novel feature of the results presented here is that two investigated lines (riT113 and riT149) were derived not by selection, but by a single temperature treatment. Finally, all the derived ri-lines, being different by expression of ri-phenotypes and by patterns of MGE localization, had similar fitnesses.

We suggest that such phenomena could take place for many other genetic systems of quantitative morphological characters of Drosophila, limited by expression of oligogenes. In this study, the correlation between genetic modification and the $D m-412$ localization sites was discovered only in the background of the oligogene mutation $r i$. In an $\mathrm{ri}^{+}$background, both selection and temperature treatment have no effect on vein length, though MGE transpositions are not excluded.

Given that wild-type alleles of other oligogenes are present, being redundant in their expression, modifier effects are invisible. Study of other oligogene mutations, particularly those affecting wing morphogenesis might discover similar phenomena. The results of early experiments of SvetLov \& KorSAKOva $(1966,1972)$ argue in favour of this supposition. They had found that the expression of oligogene mutations forked and 
eyeless of Drosophila melanogaster changed after stress heating or cooling during the sensitive periods, and the results were inherited for tens of generations.

The family of MGE $D m-412$ ( $m d g-2)$ is not the only one expressing the $r i$ character; MGEs of three other families had very similar properties (data now in preparation).

Received October 6, 1986.

Accepted September 3, 1987.

\section{Acknowledgements}

We express our gratitude to V.A. Gvozdev, E.Sp. Belyaeva and E.G. Pasyukova for preliminary investigations of our Drosophila lines; to V.A. Gvozdev, L.Z. KaIdanov, S.N. Rodin, V.F. Semeshin, V.A. Berdnikov, for interest in our work and helpful critical remarks ; to A.A. ZharkiKH, for help in treatment of experimental material.

\section{References}

Ananyev E.V., 1984. Molecular cytogenetics of mobile genetic elements of Drosophila melanogaster (in Russian). Itogy nauki i techniki, Molekularnaya Biologia, 20, 65-105, VINITI-Press, Moscow.

Birnboim H.C., Doly J.N., 1979. A rapid alkaline extraction method for screening recombinant plasmid DNA. Nucl. Acid. Res., 7, 1513.

Crow J.F., Kimura M., 1970. An introduction to population genetics theory. 431 p., Harper and Row, New York.

Gall J.G., Pardue M.L., 1971. Nucleic acid hybridization in cytological preparations. Methods Enzymol., 21, 470-480.

Georgiev G.P., Gvozdev V.A., 1980. A mobile dispersed gene of eucaryotes (in Russian). Vestn. AN SSSR, 8, 19-27.

Gvozdev V.A., Kaidanov L.Z., 1986. Genetic variability induced by transpositions of mobile elements, and the fitness of individuals of Drosophila melanogaster (in Russian). J. Obsjei Biologii, 47, 51-63.

Gvozdev V.A., Belyaeva E.Sp., Ilyin Y.N., Amosova I.S., Kaidanov L.Z., 1981. Selection and transposition of mobile dispersed genes in Drosophila melanogaster. Cold Spring Harbor Symp. Quant. Biol., 45, 673-685.

Khesin R.B., 1984. The instability of the genome (in Russian). 472 p., Nauka Publ., Moscow.

Lindsley D.L., Grell E.H., 1968. Genetic variations of Drosophila melanogaster. 472 p., Carnegie Inst. Washington Publications 627.

Maniatis T., Fritsch E.F., Sambrook J., 1982. Molecular cloning. 505 p., Cold Spring Harbor Laboratory, New York.

Mather K., Jinks J.L., 1982. Biometrical genetics. 463 p., Chapman and Hall, London.

Pasyukova E.G., Belyaeva E.Sp., Cogan G.L., Pavlov M.B., Kaidanov L.Z., Gvozdev V.A., 1984. Transposition of mobile dispersed genes, correlated with change of the fitness in Drosophila melanogaster (in Russian). Genetika, 20, 1772-1781.

Pasyukova E.G., Cogan G.L., Iovleva O.V., Kaidanov L.Z., Gvozdev V.A., 1985. Coordinated changes of location of mobile elements in the genome of Drosophila melanogaster, reflecting the results of directed selection for quantitative characters (in Russian). Doklady AN SSSR, 283, $1476-1480$. 
Ratner V.A., Vasilyeva L.A., 1987. A quantitative character in Drosophila: genetic, ontogenetic, cytogenetic and population aspects (in Russian). Genetika, 23, 1070-1081.

Rubin G.M., 1983. Dispersed repetitive DNAs in Drosophila. In : ShapIRo J. (ed.), Mobile genetic elements, 329-361, Academic Press, New York.

SNEATH P.H.A., SOKAL R.R., 1973. Numerical taxonomy: the principles and practice of numerical classification. 573 p., Freeman, San Francisco.

Svetlov P.G., Korsakova G.F., 1966. Prolonged modification of forked mutations in experiments with temperature treatment of larvae of Drosophila melanogaster (in Russian). Doklady AN SSSR, 170, 439-442.

SvetLov P.G., KoRSaKova G.F., 1972. The inheritance of changes in expressivity of Drosophila melanogaster eyeless mutations induced by temperature treatments in determinate development periods (in Russian). Ontogenes, 2, 347-355.

Urbakh V.Yu., 1964. Biometrical methods (in Russian). 360 p., Nauka Publ., Moscow.

VASILYeva L.A., 1979. Analysis of the nature of selection limits in populations under selection for a quantitative character in the case of Drosophila melanogaster populations (in Russian). Genetika, 15, 1588-1598.

VASILYEVA L.A., 1981. Analysis of a system controlling the expression of the radius incompletus mutation in Drosophila melanogaster. Abstr. of 7 th Europ. Drosophila Res. Conf., Oulu, Finland, 30 June-3 July, 1981, 104, EDRC, Oulu.

VASILYEVA L.A., 1982. An influence of temperature step-changing on the expressivity of the radius incompletus (ri) character of Drosophila melanogaster. In : Novak V.J.A., MLixovsky (ed.), Evolution and environment, 87-89, CAS, Praha.

VASILYEVA L.A., $1984 a$. Analysis of results of long term selection for a quantitative character in Drosophila melanogaster populations (in Russian). Genetika, 20, 595-598.

VASILYeva L.A., $1984 \mathrm{~b}$. Analysis of the system expressed in the wing radial vein in Drosophila melanogaster (in Russian). Genetika, 20, 599-604.

VASILYEVA L.A., $1984 c$. The temperature influence on expression of wing radial vein in Drosophila melanogaster (in Russian). J. Obsjei Biologii, 45, 859-864.

VASILYeva L.A., Nikoro Z.S., 1976. The dynamics of response to selection and analysis of causes of selection plateaux in populations (in Russian). Genetika, 12, 63-72.

Vasilyeva L.A., Mukhina L.I., 1985. The influence of genotypical environment on the expression of radial vein of the Drosophila melanogaster wing (in Russian). J. Obsjei Biologii, 46, 78-83.

Vasilyeva L.A., Zabanov S.A., 1984/1985. Evolutionary properties of the genetic system determining a quantitative character of Drosophila (in Russian). Abstr. of Ist All-Union conference on the problems of evolution, Moscow, 28-3I January, 1985, 7-8, AN SSSR, Moscow.

Vasilyeva L.A., Zabanov S.A., Ratner V.A., 1985. On the possible role of mobile genetic elements (MGE) in determination and evolution of quantitative characters. Abstr. of Internat. Symposium «Biological evolution", Bari (Italy), 9-13 April, 1985, 84-86, Universita di Bari, Bari (Italy).

Vasilyeva L.A., Ratner V.A., Zabanov S.A., 1987 a. Expression of the radius incompletus quantitative character, temperature effects and localization of the mobile elements in Drosophila. 1. The properties of subpopulations studied (in Russian). Genetika, 23, 71-80.

Vasilyeva L.A., Zabanov S.A., Ratner V.A., Zhimulev I.F., Protopopov M.O., Belyaeva E.S., $1987 \mathrm{~b}$. Expression of the radius incompletus quantitative character, temperature effects and localization of mobile elements in Drosophila (in Russian). 2. MGE Dm-412. Genetika, 23, 81-92. 